\title{
Studies on the Manifestation of Hybrid vigour and Combining Ability in Polyvoltine x Bivoltine (cross breed) Hybrids of Mulberry Silkworm Bombyx mori L.
}

\author{
S. V. Seshagiri ${ }^{1 * *}$, S. Sankar Naik ${ }^{2}$, J. Seetharamulu ${ }^{1}$, A.K. Goel ${ }^{1}$, and P. J. Raju ${ }^{1}$ \\ ${ }^{I}$ Andhra Pradesh State Sericulture Research and Development Institute, Kirikera - 515 211, Hindupur, Andhra Pradesh, India \\ ${ }^{2}$ Department of Sericulture, Sri Krishnadevaraya University, Anantapur - 515 055, Andhra Pradesh, India.
}

(Received 28 September 2011; Accepted 20 February 2012)

To meet the local requirement i.e. the tropical stress of Andhra Pradesh and marginal farmers, twenty four new hybrid combinations were tested in Line $x$ Tester method involving eight newly developed polyvoltine oval lines viz., MSO1, MSO2, MSO3, MSO4, MSO5, MSO6, MSO7 and MSO8 and three bivoltine silkworm breeds viz., APS12, APS45 and APS8 as testers. These hybrids were reared to evaluate their performance. Data for eight economically important metric traits viz., fecundity, cocoon yield per 10,000 larvae by number, cocoon yield per 10,000 larvae by weight, survival rate, cocoon weight, cocoon shell weight, cocoon shell ratio and filament length was recorded. General combining ability (gca), specific combining ability (sca) and heterosis were analyzed. Further, the relative merit of the hybrids for all the traits under study was also assessed adopting Evaluation Index method. Based on combining ability effects, heterosis and evaluation index, five hybrid combinations viz., MSO3 x APS45, MSO1 $x$ APS12, MSO7 $x$ APS8, MSO2 $x$ APS45 and MSO1 $x$ APS45 were selected for further evaluation in different seasons.

Key words: Silkworm, Hybrid combinations, Performance data, Combining ability, Evaluation index.

\footnotetext{
*To whom the correspondence addressed

Andhra Pradesh State Sericulture Research and Development Institute (APSSRDI), Kirikera - 515 211, Hindupur, Andhra Pradesh, India

Tel: +094410 26695, 918556247428 ;

E-mail: seshu_apssrdi@rediffmail.com
}

\section{Introduction}

Breeding as an important tool has been utilized by many breeders which has also played a vital role in increasing the productivity in sericulture. Aim of most of the breeding programmes is to improve the yield potential and qualitative parameters of the breeds/ hybrids over the existing ones. The silkworm, Bombyx mori L. offers one of the very important insects of choice with large number of strains which is best exemplified for utilization of heterosis by crossing them in different combinations (Datta and Nagaraju, 1987). In fact, silkworm crop is the only exception where hybrids are invariably used (Yokoyama, 1979). The systematic rearing of F1 hybrids was undertaken for the first time in Japan after establishing the superiority of hybrids (Toyama, 1905). A new era came into existence with the exploitation of 'heterosis' which has increased the productivity of silk contributing to the development and expansion of sericulture industry. However, there is always a scope of improvement of genetic traits and identification of superior hybrids through estimation of heterosis by combining ability studies (Rao et al., 1968).

The recent trend of global silk production centered mainly in tropical countries. India is the second largest producer of silk in the world next to China and more than ninety percent of the silk produced mainly by cross breeds (polyvoltine $\mathrm{x}$ bivoltine). In spite of continuous efforts for the development of sericulture through various breeding programs many hybrids have been developed by the breeders (Rao et al., 2004; Seshagiri et al., 2011), still there exists a wide gap between domestic requirement and production in India. To fulfill the gap and to face global competitiveness in silk production there is a need to develop more productive breeds or 
hybrids with quantitative and qualitative merit. Very limited number of polyvoltine cross breeds are available with all desired traits, which are not sufficient to meet the demand. The polyvoltine race, Pure Mysore (PM) is ruling the Indian Sericulture Industry for more than 50 years. At this juncture, there is a need for the development of quantitatively and qualitatively superior polyvoltine breeds and hybrids with high genetic plasticity to cater various climatic conditions of tropical countries. In the present study, an attempt was made to know the general combining ability of the new genotypes, specific combining ability and heterosis of their hybrids under Line $\mathrm{x}$ Tester programme in order to identify potential parents and to adjudicate the best hybrids for commercial exploitation at farmer's level.

\section{Materials and Methods}

In the present study, eight newly evolved polyvoltine breeds viz., MSO1, MSO2, MSO3, MSO4, MSO5,
MSO6, MSO7 and MSO8 were tested with three productive superior bivoltine breeds viz., APS12, APS45 and APS8. Twenty four hybrid combinations were prepared and tested under Line $\mathrm{x}$ Tester mating design. All the hybrid combinations along with their parents were reared in three replications following standard rearing techniques (Krishnaswami, 1973). Three hundred larvae were retained after $3^{\text {rd }}$ moult in each replication. The data pertaining to eight economic traits viz., fecundity, cocoon yield per 10,000 larvae by number, cocoon yield per 10,000 larvae by weight $(\mathrm{kg})$, survival rate $(\%)$, cocoon weight $(\mathrm{g})$, cocoon shell weight $(\mathrm{g})$, cocoon shell ratio (\%) and filament length $(\mathrm{m})$ were recorded. The data was analysed using "INDOSTAT" software package. Analysis of variance (ANOVA) was carried out to study the overall differences between genotypes. The estimates of General Combining Ability (gca), Specific Combining Ability (sca), Mid parental heterosis (MPH) and Better parental heterosis (BPH) were also calculated (Kempthorne, 1957) to understand the combining ability and heterosis.

Table 1. Mean Performance of Poly x Bivoltine hybrids of silkworm, Bombyx mori L

\begin{tabular}{|c|c|c|c|c|c|c|c|c|c|}
\hline \multirow{2}{*}{ S. No. } & \multirow{2}{*}{$\begin{array}{c}\text { Hybrid / } \\
\text { Combination }\end{array}$} & \multirow{2}{*}{$\begin{array}{l}\text { Fecundity } \\
\text { (No.) }\end{array}$} & \multicolumn{2}{|c|}{ Yield /10,000 larvae } & \multirow{2}{*}{$\begin{array}{c}\text { Pupation } \\
(\%)\end{array}$} & \multirow{2}{*}{$\begin{array}{c}\text { Cocoon } \\
\text { weight }(g)\end{array}$} & \multirow{2}{*}{$\begin{array}{l}\text { Shell weight } \\
\text { (g) }\end{array}$} & \multirow{2}{*}{$\begin{array}{c}\text { Shell ratio } \\
(\%)\end{array}$} & \multirow{2}{*}{$\begin{array}{l}\text { Filament } \\
\text { length }(\mathrm{m})\end{array}$} \\
\hline & & & No. & Wt. (kg) & & & & & \\
\hline 1 & MSO1 x APS12 & 512 & 9539 & 17.798 & 94.02 & 1.888 & 0.353 & 18.70 & 937 \\
\hline 2 & MSO1 x APS45 & 502 & 9550 & 17.753 & 94.70 & 1.890 & 0.351 & 18.59 & 923 \\
\hline 3 & MSO1 x APS8 & 481 & 9122 & 16.195 & 89.56 & 1.788 & 0.323 & 18.08 & 834 \\
\hline 4 & MSO2 $\times$ APS 12 & 469 & 9111 & 15.072 & 90.27 & 1.668 & 0.296 & 17.75 & 734 \\
\hline 5 & MSO2 x APS45 & 506 & 9533 & 17.784 & 94.45 & 1.875 & 0.351 & 18.72 & 953 \\
\hline 6 & MSO2 x APS 8 & 482 & 9267 & 16.015 & 91.92 & 1.753 & 0.297 & 16.95 & 776 \\
\hline 7 & MSO3 x APS12 & 471 & 9383 & 16.855 & 92.73 & 1.833 & 0.337 & 18.38 & 825 \\
\hline 8 & MSO3 x APS45 & 516 & 9567 & 18.324 & 94.60 & 1.945 & 0.369 & 18.97 & 979 \\
\hline 9 & MSO3 x APS 8 & 477 & 9250 & 16.243 & 90.32 & 1.765 & 0.321 & 18.17 & 805 \\
\hline 10 & MSO4 x APS12 & 457 & 9100 & 14.969 & 89.67 & 1.650 & 0.275 & 16.64 & 777 \\
\hline 11 & MSO4 x APS45 & 481 & 9222 & 15.354 & 90.88 & 1.676 & 0.277 & 16.51 & 763 \\
\hline 12 & MSO4 x APS8 & 487 & 9200 & 14.339 & 91.02 & 1.569 & 0.253 & 16.14 & 727 \\
\hline 13 & MSO5 x APS 12 & 487 & 9422 & 15.798 & 93.20 & 1.748 & 0.277 & 15.84 & 701 \\
\hline 14 & MSO5 x APS45 & 454 & 9178 & 15.900 & 90.56 & 1.736 & 0.269 & 15.49 & 734 \\
\hline 15 & MSO5 x APS8 & 498 & 9211 & 15.983 & 91.12 & 1.742 & 0.284 & 16.32 & 812 \\
\hline 16 & MSO6 x APS12 & 494 & 9111 & 16.015 & 90.53 & 1.767 & 0.284 & 16.09 & 773 \\
\hline 17 & MSO6 x APS45 & 485 & 9322 & 15.351 & 92.23 & 1.654 & 0.274 & 16.58 & 759 \\
\hline 18 & MSO6 x APS8 & 484 & 9145 & 14.642 & 90.52 & 1.610 & 0.272 & 16.88 & 740 \\
\hline 19 & MSO7 x APS12 & 498 & 9189 & 15.676 & 90.73 & 1.714 & 0.279 & 16.30 & 715 \\
\hline 20 & MSO7 x APS45 & 474 & 9256 & 15.409 & 91.25 & 1.690 & 0.276 & 16.31 & 757 \\
\hline 21 & MSO7 x APS8 & 510 & 9495 & 17.794 & 94.19 & 1.887 & 0.360 & 19.08 & 926 \\
\hline 22 & MSO8 x APS12 & 492 & 9322 & 15.527 & 92.57 & 1.675 & 0.283 & 16.90 & 761 \\
\hline 23 & MSO8 x APS45 & 487 & 9022 & 14.692 & 88.96 & 1.651 & 0.278 & 16.85 & 740 \\
\hline 24 & MSO8 x APS 8 & 473 & 9045 & 15.113 & 89.55 & 1.685 & 0.288 & 17.09 & 770 \\
\hline
\end{tabular}


Table 2. ANOVA for combining ability of genetic traits in Poly x Bivoltine hybrids of silkworm

\begin{tabular}{|c|c|c|c|c|c|c|c|c|c|}
\hline \multirow{2}{*}{ Source of Variation } & \multirow{2}{*}{ D.f. } & \multirow{2}{*}{ Fecundity } & \multicolumn{2}{|c|}{ Yield /10,000 larvae } & \multirow{2}{*}{$\begin{array}{c}\text { Survival } \\
\text { rate }\end{array}$} & \multirow{2}{*}{$\begin{array}{l}\text { Cocoon } \\
\text { weight }\end{array}$} & \multirow{2}{*}{$\begin{array}{c}\text { Shell } \\
\text { weight }\end{array}$} & \multirow{2}{*}{ Shell ratio } & \multirow{2}{*}{$\begin{array}{c}\text { Filament } \\
\text { length }\end{array}$} \\
\hline & & & No. & Wt. & & & & & \\
\hline Replications & 2 & 204.07 & 26272.46667 & 0.026 & 2.70 & 0.001 & 0.000 & 0.48 & 447.58 \\
\hline Treatments & 34 & $800.186^{* *}$ & $95155.0431 * *$ & $8.782 * *$ & $10.582 * *$ & $0.093 * *$ & $0.006^{* *}$ & $4.070 * *$ & $18011.94 * *$ \\
\hline Parents & 10 & $792.721 * *$ & $79648.897 * *$ & $7.394 * *$ & $10.451 * *$ & $0.116^{* *}$ & $0.009 * *$ & $5.561 * *$ & $2683.03 * *$ \\
\hline Parents & 1 & $689.2367^{*}$ & $479618.0525^{* *}$ & $136.051 * *$ & $46.40 * *$ & $1.328 * *$ & $0.032 * *$ & $0.659 * *$ & 1213 \\
\hline Hybrids & 23 & $808.255^{* *}$ & $85181.063 * *$ & $3.852 * *$ & $9.082 * *$ & $0.030 * *$ & $0.004 * *$ & $3.570 * *$ & $55^{* *}$ \\
\hline Lines & 7 & $488.046^{*}$ & $96518.064 * *$ & $7.156^{*}$ & $7.941 * *$ & $0.060 *$ & $0.008^{*}$ & & 29119.82 \\
\hline Testers & 2 & 67.17 & 77934.59722 & $1.674 * *$ & 7.62 & $0.009^{*}$ & $0.0003 * *$ & & 14799.50 \\
\hline Line $*$ Tester & 14 & $1074.2301 * *$ & $80547.772 * *$ & $2.511 * *$ & $9.860 * *$ & $0.018 * *$ & $0.0019 * *$ & $1.722 * *$ & $16482.91 * *$ \\
\hline Error & 68 & 107.27 & 12317.46667 & 0.03 & 1.32 & 0.000 & 0.000 & 0.08 & 221.07 \\
\hline
\end{tabular}

* Significant $(\mathrm{P}<0.05)$; ** Significant $(\mathrm{P}<0.01)$

Table 3. Percent contribution for genetic traits in silkworm, Bombyx mori L.

\begin{tabular}{cccccccccc}
\hline \multirow{2}{*}{ Source } & \multirow{2}{*}{ Fecundity } & \multicolumn{2}{c}{ Yield per 10,000 Larvae } & $\begin{array}{c}\text { Survival } \\
\text { Rate }\end{array}$ & $\begin{array}{c}\text { Cocoon } \\
\text { Weight }\end{array}$ & Shell Weight & $\begin{array}{c}\text { Shell Ratio } \\
\text { No. }\end{array}$ & Wt. $(\mathrm{Kg})$ & $\begin{array}{c}\text { Filament } \\
\text { Length }\end{array}$ \\
\hline Lines & 78.38 & 34.48 & 46.54 & 66.61 & 31.25 & 31.78 & 29.36 & 43.91 \\
Testers & 10.90 & 7.96 & 3.78 & 26.09 & 22.70 & 55.36 & 59.88 & 6.38 \\
Lines x Testers & 10.72 & 57.56 & 49.68 & 7.30 & 46.05 & 12.86 & 10.76 & 49.71 \\
\hline
\end{tabular}

\section{Multiple Trait Evaluation Index Method}

Evaluation index over multiple traits was calculated for each hybrid for all the traits as per the following formula which is suggested by Mano et al. (1993).

$$
\text { Evaluation Index }=\frac{\mathrm{A}-\mathrm{B}}{\mathrm{C}} \times 10+50
$$

Where,

$A=$ Value obtained for a trait by the specific hybrid

$\mathrm{B}=$ Overall mean value of particular trait over all the hybrids

$\mathrm{C}=$ Standard deviation of the trait over all the hybrids

$10=$ Constant used for change of scale

$50=$ Constant used for change of origin

Based on the average Evaluation Index value ranks were assigned to all the hybrid combinations. Evaluation Index, number of traits with positive SCA, number of traits with positive mid parent heterosis (MPH), number of traits with positive better parent heterosis $(\mathrm{BPH})$ and total score of positive SCA, MPH and BPH exhibited by each of the twenty four hybrid combinations were compiled for ultimate identification of promising hybrids (Goel, 2008).

\section{Results}

The mean rearing performance data and ANOVA per- taining to eight economic traits of the twenty four hybrid combinations are presented in Table 1 and 2 respectively. Perusal of the data revealed that fecundity ranged from 454 eggs/laying (MSO5 x APS45) to 516 eggs/laying (MSO3 x APS45), yield per 10000 larvae by number varied from a minimum of 9022 (MSO8 x APS45) to a maximum 9567 (MSO3 x APS45), minimum yield per 10000 larvae by weight of $14.339 \mathrm{~kg}$ was recorded in MSO4 x APS8 with a maximum of 18.324 $\mathrm{kg}$ in MSO3 $\mathrm{x}$ APS45, survival rate ranged from $88.96 \%$ (MSO8 x APS45) to $94.70 \%$ (MSO1 x APS45), Cocoon weight varied from $1.569 \mathrm{~g}$ (MSO4 x APS8) to $1.945 \mathrm{~g}$ (MSO3 x APS45), Shell weight ranged from $0.253 \mathrm{~g}$ (MSO4 x APS8) to $0.369 \mathrm{~g}$ (MSO3 x APS45) and the Shell ratio was recorded to be minimum in MSO5 x APS45 (15.49\%) with a maximum of 19.08\% in MSO7 x APS8. The Filament length varied from a minimum of $701 \mathrm{~m}$ (MSO5 $\mathrm{x}$ APS12) to a maximum of $979 \mathrm{~m}$ (MSO3 x APS45). The ANOVA for combining ability revealed significant differences for general combining ability (gca) and specific combining ability (sca) indicating the genetic diversity of the parental breeds/ hybrids.

\section{Contribution for genetic traits}

The data (Table 3 ) revealed that contribution of lines was more for the traits of fecundity $(78.38 \%)$ and survivability $(66.61 \%)$ where as testers contribution was 
Table 4. General combining ability effects of lines and testers

\begin{tabular}{|c|c|c|c|c|c|c|c|c|}
\hline \multirow{2}{*}{ Parents } & \multirow{2}{*}{ Fecundity } & \multicolumn{2}{|c|}{ Yield per 10,000 Larvae } & \multirow{2}{*}{-Survival Rate } & \multirow{2}{*}{$\begin{array}{l}\text { Cocoon } \\
\text { Weight }\end{array}$} & \multirow{2}{*}{ Shell Weight } & \multirow{2}{*}{ Shell Ratio } & \multirow{2}{*}{$\begin{array}{c}\text { Filament } \\
\text { Length }\end{array}$} \\
\hline & & No. & Wt. & & & & & \\
\hline \multicolumn{9}{|l|}{ Lines } \\
\hline MSO1 & $12.208 * *$ & $129.778 * *$ & $1.218 * *$ & $1.076 * *$ & $0.111 * *$ & $0.041 * *$ & $1.228 * *$ & $98.125 * *$ \\
\hline $\mathrm{MSO} 2$ & -0.903 & 15.000 & $0.270 * *$ & 0.33 & $0.023 * *$ & $0.014 * *$ & $0.568 * *$ & $18.681 * *$ \\
\hline MSO3 & 0.319 & $140.889 * *$ & $1.138 * *$ & $1.249 * *$ & $0.103 * *$ & $0.041 * *$ & $1.272 * *$ & $66.014 * *$ \\
\hline MSO4 & -10.792 & -96.111 & -1.139 & -1.053 & -0.113 & -0.032 & -0.732 & -44.764 \\
\hline MSO5 & -6.903 & -3.444 & -0.137 & -0.06 & -0.002 & -0.025 & -1.344 & -51.208 \\
\hline MSO6 & 1.208 & -81.222 & -0.694 & -0.59 & -0.067 & -0.024 & -0.709 & -42.542 \\
\hline MSO7 & $7.542 *$ & 39.222 & $0.263 * *$ & 0.372 & $0.019 * *$ & $0.004 *$ & 0.002 & -0.986 \\
\hline MSO8 & -2.681 & -144.111 & -0.92 & -1.323 & -0.074 & -0.018 & -0.284 & -43.319 \\
\hline $\mathrm{SE}$ & 3.452 & 36.995 & 0.060 & 0.382 & 0.003 & 0.002 & 0.095 & 4.956 \\
\hline $\mathrm{CD}$ at $5 \%$ & 6.949 & 74.466 & 0.122 & 0.769 & 0.006 & 0.003 & 0.191 & 9.976 \\
\hline $\mathrm{CD}$ at $1 \%$ & 9.277 & 99.405 & 0.163 & 1.027 & 0.008 & 0.005 & 0.255 & 13.317 \\
\hline \multicolumn{9}{|l|}{ Testers } \\
\hline APS12 & -1.750 & -0.931 & -0.065 & $0.077^{*}$ & -0.002 & -0.003 & -0.124 & -23.583 \\
\hline APS45 & 1.583 & $57.444 *$ & $0.291 * *$ & $0.521 *$ & $0.020 * *$ & $0.004 * *$ & 0.024 & $25.917 * *$ \\
\hline APS8 & 0.167 & -56.514 & -0.225 & -0.598 & -0.019 & -0.002 & 0.100 & -2.333 \\
\hline SE & 2.114 & 22.654 & 0.037 & 0.234 & 0.002 & 0.001 & 0.058 & 3.035 \\
\hline $\mathrm{CD}$ at $5 \%$ & 4.256 & 45.601 & 0.075 & 0.471 & 0.004 & 0.002 & 0.117 & 6.109 \\
\hline $\mathrm{CD}$ at $1 \%$ & 5.681 & 60.873 & 0.100 & 0.629 & 0.005 & 0.003 & 0.156 & 8.155 \\
\hline
\end{tabular}

* Significant $(\mathrm{P}<0.05)$; ** Significant $(\mathrm{P}<0.01)$

Table 5. Specific combining ability effects in Poly $\mathrm{x}$ Bivoltine hybrids of silkworm for genetic traits

\begin{tabular}{|c|c|c|c|c|c|c|c|c|}
\hline \multirow{2}{*}{ Hybrid combination } & \multirow{2}{*}{ Fecundity } & \multicolumn{2}{|c|}{ Yield per 10,000 Larvae } & \multirow{2}{*}{ Pupation } & \multirow{2}{*}{$\begin{array}{l}\text { Cocoon } \\
\text { Weight }\end{array}$} & \multirow{2}{*}{ Shell Weight } & \multirow{2}{*}{ Shell Ratio } & \multirow{2}{*}{$\begin{array}{l}\text { Filament } \\
\text { Length }\end{array}$} \\
\hline & & No. & Wt. & & & & & \\
\hline MSO1 x APS12 & $15.417^{*}$ & $136.264 *$ & $0.614 * *$ & 1.186 & $0.034 * *$ & $0.013 * *$ & $0.372 *$ & $62.583 * *$ \\
\hline MSO1 x APS45 & 2.083 & 88.889 & $0.214 *$ & $1.419 *$ & $0.015 * *$ & 0.004 & 0.103 & -0.917 \\
\hline MSO1 x APS8 & -17.500 & -225.153 & -0.828 & -2.605 & -0.049 & -0.018 & -0.476 & -61.667 \\
\hline $\mathrm{MSO} 2 \times \mathrm{APS} 12$ & -14.806 & -176.625 & -1.163 & -1.822 & -0.097 & -0.016 & 0.076 & -61.639 \\
\hline $\mathrm{MSO} 2 \times \mathrm{APS} 45$ & $18.861 * *$ & $187.000 * *$ & $1.193 *$ & $1.915 * *$ & $0.088 * *$ & $0.032 * *$ & $0.897 * *$ & $108.194 * *$ \\
\hline MSO2 x APS8 & -4.056 & -10.375 & -0.03 & -0.093 & 0.010 & -0.016 & -0.972 & -46.556 \\
\hline MSO3x APS12 & -18.694 & -36.181 & -0.249 & & -0.015 & -0.002 & & -26.639 \\
\hline MSO3 x APS45 & $27.972 * *$ & 94.444 & $0.865 * *$ & & $0.078 * *$ & $0.023 * *$ & $0.446 * *$ & $86.861 * *$ \\
\hline MSO3 x APS 8 & -9.278 & -58.264 & -0.616 & -0.922 & -0.063 & -0.02 & -0.48 & -60.222 \\
\hline MSO4 x APS12 & -14.917 & -65.847 & 0.155 & -0.715 & $0.019 * *$ & $0.011 * *$ & $0.462 * *$ & $44.472 * *$ \\
\hline MSO4 x APS45 & 3.417 & -12.889 & 0.172 & -0.269 & $0.025 * *$ & 0.003 & -0.003 & -18.028 \\
\hline MSO4 x APS8 & 11.5 & 78.736 & -0.327 & 0.984 & -0.044 & -0.014 & -0.459 & -26.444 \\
\hline MSO5 x APS12 & 8.861 & $152.819 *$ & -0.031 & $1.498 *$ & 0.008 & 0.002 & 0.081 & -24.75 \\
\hline MSO5 x APS45 & -27.472 & -149.889 & -0.284 & -1.589 & -0.027 & -0.012 & -0.421 & -40.583 \\
\hline MSO5x APS8 & $18.611 * *$ & -2.931 & $0.315 *$ & 0.090 & $0.019 * *$ & $0.009 * *$ & $0.340 *$ & $65.333 * *$ \\
\hline MSO6 x APS12 & 8.417 & -80.736 & $0.744 * *$ & -0.645 & $0.092 * *$ & $0.010 * *$ & -0.301 & $39.250 * *$ \\
\hline MSO6 x APS45 & -4.25 & 72.222 & -0.276 & 0.618 & -0.043 & -0.007 & 0.04 & -24.25 \\
\hline MSO6 x APS8 & -4.167 & 8.514 & -0.468 & 0.027 & -0.049 & -0.004 & 0.261 & -15 \\
\hline MSO7 x APS12 & 5.75 & -123.181 & -0.552 & -1.407 & -0.048 & -0.023 & -0.802 & -60.639 \\
\hline MSO7 x APS45 & -21.917 & -114.889 & -1.175 & -1.324 & -0.094 & -0.034 & -0.941 & -68.139 \\
\hline
\end{tabular}


Table 5. Continued

\begin{tabular}{|c|c|c|c|c|c|c|c|c|}
\hline \multirow{2}{*}{ Hybrid combination } & \multirow{2}{*}{ Fecundity } & \multicolumn{2}{|c|}{ Yield per 10,000 Larvae } & \multirow{2}{*}{ Pupation } & \multirow{2}{*}{$\begin{array}{l}\text { Cocoon } \\
\text { Weight }\end{array}$} & \multirow{2}{*}{ Shell Weight } & \multirow{2}{*}{ Shell Ratio } & \multirow{2}{*}{$\begin{array}{c}\text { Filament } \\
\text { Length }\end{array}$} \\
\hline & & No. & Wt. & & & & & \\
\hline MSO7 x APS8 & $16.167 * *$ & $238.069 * *$ & $1.727 * *$ & $2.732 * *$ & $0.142 * *$ & $0.056 * *$ & $1.743 * *$ & $128.778 * *$ \\
\hline MSO8 $\times$ APS 12 & 9.972 & $193.486 * *$ & $0.481 * *$ & $2.132 * *$ & 0.007 & 0.003 & 0.078 & $27.361 * *$ \\
\hline MSO8 x APS45 & 1.306 & -164.889 & -0.709 & -1.919 & -0.04 & -0.009 & -0.121 & -43.139 \\
\hline MSO $8 \times$ APS 8 & -11.278 & -28.597 & $0.228 *$ & -0.213 & $0.033 * *$ & $0.006 *$ & 0.043 & 15.778 \\
\hline Std. Error & 5.980 & 64.077 & 0.105 & 0.662 & 0.054 & 0.003 & 0.164 & 8.584 \\
\hline $\mathrm{CD}$ at $5 \%$ & 12.04 & 128.979 & 0.211 & 1.333 & 0.011 & 0.006 & 0.330 & 17.279 \\
\hline $\mathrm{CD}$ at $1 \%$ & 16.07 & 172.174 & 0.282 & 1.779 & 0.014 & 0.008 & 0.441 & 23.066 \\
\hline
\end{tabular}

* Significant $(\mathrm{P}<0.05)$;** Significant $(\mathrm{P}<0.01)$

Table 6. Mid parent heterosis (MPH) among Poly x Bivoltine hybrids for various genetic traits

\begin{tabular}{|c|c|c|c|c|c|c|c|c|}
\hline \multirow{2}{*}{ Hybrid combination } & \multirow{2}{*}{ Fecundity } & \multicolumn{2}{|c|}{ Yield per 10,000 Larvae } & \multirow{2}{*}{ Pupation } & \multirow{2}{*}{$\begin{array}{l}\text { Cocoon } \\
\text { Weight }\end{array}$} & \multirow{2}{*}{ Shell Weight } & \multirow{2}{*}{ Shell Ratio } & \multirow{2}{*}{$\begin{array}{c}\text { Filament } \\
\text { Length }\end{array}$} \\
\hline & & No. & Wt. & & & & & \\
\hline MSO1 x APS12 & $3.40^{*}$ & $4.76^{* *}$ & $24.57 * *$ & $5.02 * *$ & $18.19 * *$ & $18.66^{* *}$ & 1.53 & $23.69 * *$ \\
\hline MSO1 x APS45 & 2.45 & $4.81 * *$ & $23.27 * *$ & $5.28 * *$ & $17.81 * *$ & $19.50 * *$ & $2.38 *$ & $23.55 * *$ \\
\hline MSO1 x APS8 & -0.48 & 0.86 & $14.12 * *$ & -0.08 & $12.29 * *$ & $12.33 * *$ & 0.77 & $16.07 * *$ \\
\hline MSO2 x APS12 & -4.9 & 0.25 & $5.12 * *$ & 0.98 & $4.45 * *$ & 0.62 & -2.4 & -2.72 \\
\hline $\mathrm{MSO} 2 \times \mathrm{APS} 45$ & $3.69^{*}$ & $4.82 * *$ & $23.06^{* *}$ & $5.16^{* *}$ & $16.89 * *$ & $20.76^{* *}$ & $4.48 * *$ & $28.15^{* *}$ \\
\hline MSO2 x APS 8 & 0.07 & $2.15^{*}$ & $12.66^{* *}$ & $2.04 *$ & $10.46^{* *}$ & $4.63 * *$ & -4.41 & $7.67 * *$ \\
\hline MSO3x APS12 & -5.38 & $2.74 * *$ & $16.33^{* *}$ & $3.29 * *$ & $14.00 * *$ & $12.47 * *$ & -0.27 & $6.92 * *$ \\
\hline MSO3 x APS45 & $5.88 * *$ & $4.74 * *$ & $25.48 * *$ & $4.83 * *$ & $20.63 * *$ & $24.73 * *$ & $4.29 * *$ & $30.07 * *$ \\
\hline MSO3 x APS 8 & -0.69 & $2.57 * *$ & $13.44 * *$ & 1.66 & $10.34 * *$ & $10.47 * *$ & 0.78 & $10.98 * *$ \\
\hline MSO4 x APS12 & -6.39 & 0.80 & $5.61 * *$ & 1.3 & $3.81 * *$ & -5.02 & -7.2 & $4.74 * *$ \\
\hline MSO4 x APS45 & -0.93 & $1.97 *$ & $7.37 * *$ & 1.82 & $5.09 * *$ & -4.1 & -7.57 & $4.47 * *$ \\
\hline MSO4 x APS8 & 1.85 & $2.47 * *$ & 1.76 & $2.33^{*}$ & -0.86 & -10.39 & -8.63 & $3.49 *$ \\
\hline MSO5 x APS 12 & -0.88 & $3.79 * *$ & $10.45^{* *}$ & $4.34 * *$ & $9.76^{* *}$ & -5.84 & -13.13 & -6.03 \\
\hline MSO5 x APS45 & -6.62 & 1.04 & $10.28 * *$ & 0.91 & $8.47 * *$ & -7.57 & -13.8 & -0.11 \\
\hline MSO5x APS8 & $4.00^{*}$ & $2.15^{*}$ & $12.49 * *$ & $1.90^{*}$ & $9.74 * *$ & -0.06 & -8.06 & $14.91 * *$ \\
\hline MSO6 x APS12 & 0.34 & 0.52 & $11.85^{* *}$ & 1.63 & $10.77 * *$ & -3.07 & -11.3 & 2.23 \\
\hline MSO6 x APS45 & -0.51 & $2.79 * *$ & $6.37 * *$ & $3.06^{* *}$ & $3.21 * *$ & -5.35 & -7.22 & 1.79 \\
\hline MSO6 x APS8 & 0.59 & 1.57 & $2.95 * *$ & 1.51 & $1.24 * *$ & -4.23 & -4.43 & $3.18^{*}$ \\
\hline MSO7 x APS12 & -0.66 & 0.55 & $7.80 * *$ & 0.92 & $6.43 * *$ & -6.52 & -11.33 & -5.9 \\
\hline MSO7 x APS45 & -4.53 & 1.21 & $5.14 * *$ & 1.04 & $4.46^{* *}$ & -6.76 & -9.95 & 1.02 \\
\hline MSO7 x APS8 & $4.26^{* *}$ & $4.59 * *$ & $23.17 * *$ & $4.66^{* *}$ & $17.57 * *$ & $24.35^{* *}$ & $6.52 * *$ & $28.42 * *$ \\
\hline MSO8 x APS 12 & 0.17 & $3.65 * *$ & $9.72 * *$ & $4.78 * *$ & $5.44 * *$ & -4.23 & -8.07 & 1.72 \\
\hline MSO8 x APS45 & 0.14 & 0.25 & $2.99 * *$ & 0.22 & $3.41 * *$ & -4.79 & -6.98 & 0.32 \\
\hline MSO8 x APS8 & -1.39 & 1.25 & $7.52 * *$ & 1.25 & $6.40 * *$ & 0.64 & -4.54 & $8.68 * *$ \\
\hline
\end{tabular}

* Significant $(\mathrm{P}<0.05)$; ** Significant $(\mathrm{P}<0.01)$

recorded to be higher for shell weight $(55.36 \%)$ and shell ratio $(59.88 \%)$. The interaction of lines $\mathrm{x}$ testers contributed more for the traits of yield per 10000 larvae by number $(57.56 \%)$, yield per 10000 larvae by weight $(49.68 \%)$, cocoon weight $(46.05 \%)$ and filament length $(49.71 \%)$.
General Combining Ability (gca) and Specific Combining Ability (sca)

The gca data (Table 4) revealed that the line MSO1 exhibited significant gca effects for all the traits studied followed by MSO3 for seven traits except fecundity. Among testers only two testers recorded significant positive gca effects. 
Table 7. Better parent heterosis (BPH) in silkworm hybrid combinations for the quantitative genetic traits

\begin{tabular}{|c|c|c|c|c|c|c|c|c|}
\hline \multirow{2}{*}{ Hybrid combination } & \multirow{2}{*}{ Fecundity } & \multicolumn{2}{|c|}{ Yield per 10,000 Larvae } & \multirow{2}{*}{ Pupation } & \multirow{2}{*}{$\begin{array}{l}\text { Cocoon } \\
\text { Weight }\end{array}$} & \multirow{2}{*}{ Shell Weight } & \multirow{2}{*}{ Shell Ratio } & \multirow{2}{*}{$\begin{array}{c}\text { Filament } \\
\text { Length }\end{array}$} \\
\hline & & No. & Wt. & & & & & \\
\hline MSO1 x APS12 & -0.39 & $3.00 * *$ & $11.37 * *$ & $2.52 *$ & $4.50 * *$ & -2.13 & -6.31 & $18.45 * *$ \\
\hline MSO1 x APS45 & -0.33 & $3.12 * *$ & $9.53 * *$ & $3.26 * *$ & $3.81 * *$ & 0.66 & -4.32 & $19.86 * *$ \\
\hline MSO1 x APS8 & -1.9 & -1.5 & $2.57 * *$ & -2.35 & -0.46 & -5.27 & -4.86 & $15.19 * *$ \\
\hline MSO2 x APS 12 & -8.81 & -1.26 & -5.69 & -1.29 & -7.66 & -17.93 & -11.1 & -7.29 \\
\hline MSO2 x APS45 & 0.40 & $3.31 * *$ & $9.72 * *$ & $3.28 * *$ & $2.98 * *$ & 0.75 & -3.64 & $23.71 * *$ \\
\hline MSO2 x APS8 & -1.83 & -0.07 & 1.62 & -0.14 & -2.1 & -12.79 & -10.95 & $7.39 * *$ \\
\hline MSO3x APS12 & -9.33 & 0.77 & $5.47 * *$ & 0.52 & $1.29 * *$ & -6.65 & -7.78 & $3.12 *$ \\
\hline MSO3 x APS45 & 2.45 & $2.80 * *$ & $13.05 * *$ & $2.49 *$ & $6.81 * *$ & $4.34 * *$ & -2.33 & $27.09 * *$ \\
\hline MSO3 x APS8 & -2.65 & -0.06 & $3.40 * *$ & -0.96 & -1.71 & -6.25 & -4.65 & $9.34 * *$ \\
\hline MSO4 x APS12 & -10.76 & -0.18 & -6.25 & -0.38 & -8.77 & -23.11 & -15.68 & -1.9 \\
\hline MSO4 x APS45 & -4.63 & 1.04 & -5.27 & 0.61 & -7.96 & -21.77 & -14.96 & -0.91 \\
\hline MSO4 x APS8 & -0.68 & 0.79 & -9.19 & 0.76 & -12.66 & -25.88 & -15.09 & 1.87 \\
\hline MSO5 x APS 12 & -5.38 & $2.35 *$ & -1.15 & $2.09 *$ & -3.21 & -23.2 & -20.65 & -11.46 \\
\hline MSO5 x APS45 & -9.99 & -0.3 & -1.9 & -0.81 & -4.69 & -24.03 & -20.27 & -4.67 \\
\hline MSO5x APS8 & 1.56 & 0.06 & 1.22 & -0.19 & -2.99 & -16.7 & -14.1 & $13.83 * *$ \\
\hline MSO6 x APS12 & -3.89 & -0.73 & 0.21 & -0.31 & -2.16 & -21.16 & -19.39 & -2.27 \\
\hline MSO6 x APS45 & -3.77 & 1.57 & -5.29 & 1.57 & -9.15 & -22.43 & -14.62 & -1.43 \\
\hline MSO6 x APS8 & -1.43 & -0.36 & -7.27 & -0.31 & -10.36 & -20.41 & -11.17 & 2.59 \\
\hline MSO7 x APS12 & -3.18 & -1.49 & -1.91 & -1.86 & -5.13 & -22.46 & -18.33 & -9.65 \\
\hline MSO7 x APS45 & -6.02 & -0.78 & -4.94 & -1.29 & -7.21 & -22.05 & -16.01 & -1.73 \\
\hline MSO7 x APS8 & $4.01 *$ & 1.79 & $12.69 * *$ & 1.88 & $5.07 * *$ & $5.47 * *$ & 0.37 & $27.09 * *$ \\
\hline MSO8 x APS12 & -4.34 & $3.15 * *$ & -2.84 & $3.60 * *$ & -7.25 & -21.53 & -15.36 & -3.88 \\
\hline MSO8 x APS45 & -3.44 & -0.17 & -9.36 & -0.43 & -9.35 & -21.39 & -13.27 & -3.98 \\
\hline MSO8 x APS8 & -3.67 & 0.08 & -4.29 & 0.22 & -6.18 & -15.72 & -10.09 & 7.99 \\
\hline
\end{tabular}

Significant $(\mathrm{P}<0.05) ; * *$ Significant $(\mathrm{P}<0.01)$

The tester APS45 exhibited significant gca effects for six out of eight traits studied followed by APS12, which exhibited significant positive gca effect for survival rate only.

The magnitude of sca effects (Table 5) varied considerably among hybrid combinations exhibiting both positive and negative effects indicating the genetic diversity of the parental lines. Five hybrids viz., MSO3 x APS45, MSO1 x APS12, MSO7 x APS8, MSO2 x APS45 and MSO1 x APS45 expressed significant sca effects for all the traits studied followed by MSO1 x APS45 and MSO5 $\mathrm{x}$ APS8 for seven traits.

\section{Mid parent heterosis (MPH) and Better Parent Heterosis (BPH)}

The expression of MPH (Table 6) among 24 Poly x Bivoltine hybrids varied to a greater extent and majority of the hybrids expressed significant hybrid vigour for yield per 10000 larvae by number, yield per 10000 larvae by weight, cocoon weight and filament length. Five hybrids viz., MSO3 x APS45, MSO1 x APS12, MSO7 x APS8,
MSO2 x APS45 and MSO1 x APS45 manifested significant MPH for all the eight traits studied followed by two hybrids for seven traits and eight hybrids with significant positive MPH for six traits.

The BPH (Table 7) among 24 hybrid combinations varied to a greater extent. The majority of the hybrids expressed significant BPH for yield per 10000 larvae by number, yield per 10000 larvae by weight, cocoon weight and filament length. Two hybrids viz., MSO3 x APS45 and MSO7 x APS8 manifested significant BPH for all the eight traits studied followed by one hybrid (MSO2 $\mathrm{x}$ APS45) for six traits.

\section{Evaluation Index (EI)}

The evaluation indices of the hybrid combinations (Table 8) for eight traits revealed that among the hybrid, MSO3 $\mathrm{x}$ APS45 ranked on top with a highest EI of 69.37 followed by MSO1 x APS12 (65.50), MSO7 x APS8 (65.42), MSO2 x APS45 (65.28), MSO1 x APS45 (64.85), MSO3 x APS12 (55.57), MSO3 x APS8 (50.54) 
Table 8. Evaluation index (EI) values of Poly x Bivoltine hybrids

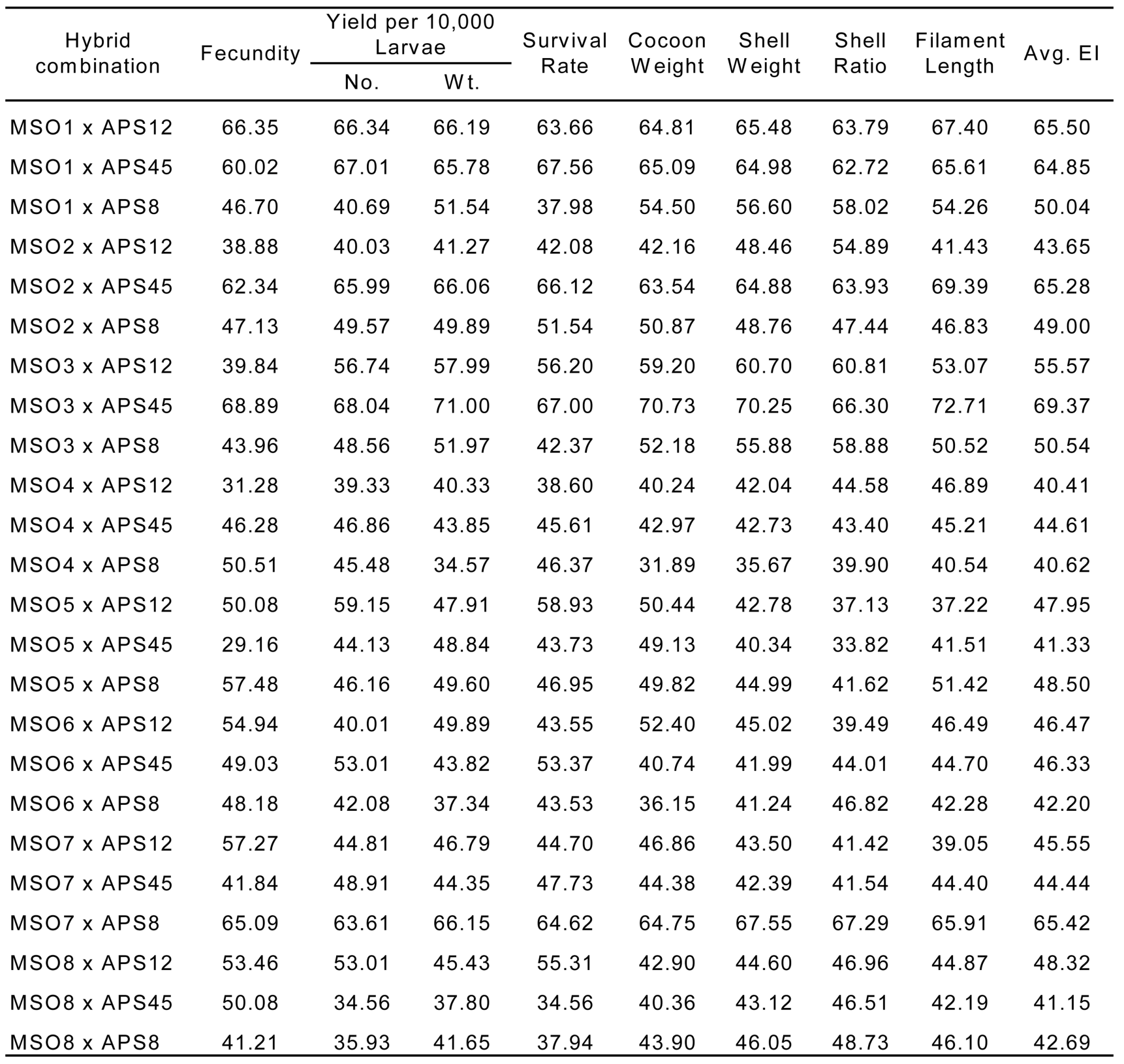

and MSO1 x APS8 (50.04).

\section{Selection of Promising Hybrid Combinations:}

On the basis of number of traits with positive of specific combining ability (sca), Mid Parent Heterosis (MPH), Better Parent Heterosis (BPH) and average evaluation index value scored by each of the twenty four hybrid combinations were reviewed (Table 9) to finally select the promising hybrids. Maximum total score of 24 positive traits for SCA, MPH and BPH was observed in two hybrids viz., MSO3 x APS45 (EI - 69.37; Score -24) and MSO7 x APS8
(EI - 65.62; Score -24) followed by MSO2 x APS45 (EI 69.37; Score - 22), MSO1 x APS12 (EI - 65.50; Score-21) and MSO1 x APS45 (EI - 64.85; Score-20). These hybrids were adjudicated as promising and chosen for further laboratory evaluation during different seasons for subsequent identification of most promising and stable combinations.

\section{Discussion}

In the present study, analysis of variance computed for 
Table 9. Selection of promising hybrid combinations based on SCA, MPH, BPH and EI

\begin{tabular}{|c|c|c|c|c|c|c|}
\hline \multirow{2}{*}{ Hybrid combination } & \multicolumn{3}{|c|}{ Total number of positive traits obtained } & \multirow{2}{*}{ Total Score } & \multirow{2}{*}{$\begin{array}{c}\text { Average } \\
\text { EI }\end{array}$} & \multirow{2}{*}{ Rank } \\
\hline & SCA & $\mathrm{MPH}$ & $\mathrm{BPH}$ & & & \\
\hline MSO1 x APS12 & 8 & 8 & 5 & 21 & 65.50 & 2 \\
\hline MSO1 x APS45 & 7 & 8 & 5 & 20 & 64.85 & 5 \\
\hline MSO1 x APS8 & 0 & 6 & 2 & 8 & 50.04 & 8 \\
\hline MSO2 x APS 12 & 1 & 5 & 0 & 6 & 43.65 & 18 \\
\hline MSO2 $\times$ APS45 & 8 & 8 & 6 & 22 & 65.28 & 4 \\
\hline MSO2 x APS 8 & 1 & 7 & 2 & 10 & 49.00 & 9 \\
\hline MSO3 x APS12 & 1 & 6 & 5 & 12 & 55.57 & 6 \\
\hline MSO3 $\times$ APS45 & 8 & 8 & 8 & 24 & 69.37 & 1 \\
\hline MSO3 x APS8 & 0 & 7 & 2 & 9 & 50.54 & 7 \\
\hline MSO4 x APS12 & 5 & 5 & 0 & 10 & 40.41 & 24 \\
\hline MSO4 x APS45 & 4 & 5 & 2 & 11 & 44.61 & 16 \\
\hline MSO4 x APS8 & 3 & 5 & 3 & 11 & 40.62 & 23 \\
\hline MSO5 x APS 12 & 6 & 4 & 2 & 12 & 47.95 & 12 \\
\hline MSO5 x APS45 & 0 & 4 & 1 & 5 & 41.33 & 21 \\
\hline MSO5 x APS8 & 7 & 6 & 4 & 17 & 48.50 & 10 \\
\hline MSO6 x APS12 & 5 & 6 & 1 & 12 & 46.47 & 13 \\
\hline MSO6 x APS45 & 3 & 5 & 2 & 10 & 46.33 & 14 \\
\hline MSO6 x APS8 & 3 & 6 & 1 & 10 & 42.20 & 20 \\
\hline MSO7 x APS12 & 1 & 4 & 0 & 5 & 45.55 & 15 \\
\hline MSO7 x APS45 & 0 & 5 & 0 & 5 & 44.44 & 17 \\
\hline MSO7 x APS8 & 8 & 8 & 8 & 24 & 65.62 & 3 \\
\hline MSO8 x APS 12 & 8 & 6 & 2 & 16 & 48.32 & 11 \\
\hline MSO8 x APS45 & 2 & 6 & 0 & 8 & 41.15 & 22 \\
\hline MSO8 x APS8 & 5 & 6 & 3 & 14 & 42.69 & 19 \\
\hline
\end{tabular}

$\mathrm{SCA}=$ Specific Combining Ability; MPH= Mid Parent Heterosis; $\mathrm{BPH}=$ Better Parent Heterosis; EI= Evaluation Index

combining ability revealed that highly significant variances due to crosses, lines and lines $\mathrm{x}$ testers observed among lines indicating the presence of both additive and non-additive gene action for the expression of these traits and also establishes the existence of genotypic differences for all the traits. In the present study, the contribution of lines was recorded to be higher for the traits viz., fecundity, survivability, which indicates the maternal effect on the traits. The testers contribution was observed to be more for the traits of shell weight and shell ratio. The interaction of lines $\mathrm{x}$ testers together contributed more for the traits like yield per 10000 larvae by number, yield per 10000 larvae by weight, cocoon weight and filament length. The results are in conformity with that of Ragavendra Rao et al. (2002).

The parental lines possessing high general combining ability (gca) are preferred for population development and for initiation of pedigree breeding as it is heritable which can be fixed. The general combining ability effects of eight lines and three testers pertaining to eight traits revealed that the line MSO1 was found to be best general combiner by virtue of significant positive effects for all the traits studied followed by MSO3 for seven traits. Among testers, APS45 exhibited significant gca effects for six out of eight traits studied indicating major role of additive gene action for the expression of these traits. The positive gca indicate their superiority and ability to combine with most of the breeds and are expected to produce promising hybrids. The identification of lines, MSO1 and MSO3 based on gca effects corroborate with the findings of Lakshmi (2008) and Goel (2008).

The magnitude of specific combining ability is another aspect to be evaluated in the hybrids to estimate their superiority. In the present investigation the sca effects varied considerably among $\mathrm{F}_{1}$ hybrids. The sca effects of the hybrids revealed the importance of both additive and non- 
additive gene action for the eight traits studied. Among the twenty four $\mathrm{F}_{1}$ hybrids analyzed, five hybrids viz., MSO3 x APS45, MSO1 x APS12, MSO7 x APS8, MSO2 $x$ APS45 and MSO1 x APS45 recorded significant positive sca effects for all the traits under study reflecting the interaction of alleles, additive and non additive gene action and dominant effects expressed by genes, which play major role in the expression of hybrid vigour which is in conformity with the studies of Goel (2008), Malik et al. (1998), Sudhakara Rao et al. (2005). The present study support the predominant role of non-additive gene action for the traits fecundity, cocoon yield per 10,000 larvae by number, cocoon yield per 10,000 larvae by weight, survival rate, cocoon weight, shell weight and cocoon shell ratio in majority of the combinations as observed by Ravindra Singh et al. (2005).

The operation of additive $\mathrm{x}$ dominance interaction in the derived hybrids showing significant sca effects involving good $\mathrm{x}$ poor general combiners is well established as in the case of MSO1 x APS12 where in, MSO1 a good combiner while APS12 is a poor combiner. In contrast to gca effects, which are related to additive $\mathrm{x}$ additive gene interaction which reflects flexible genetic variation (Griffing, 1956). The sca effects related to dominance and epistasis components of variance represents non-flexible genetic variation in nature (Simmonds, 1979).

The exploitation of heterosis is an important step towards achieving desirable economic effects in the hybrids. The hybrid combination MSO3 x APS45 recorded highly significant positive $(\mathrm{P}<0.01)$ desirable mid parent and better parent heterosis for eight and five traits respectively followed by MSO1 x APS12 with significant positive MPH for seven traits and BPH for five traits. The present findings are in agreement with Nanjundaswamy (1997). The hybrids derived in the present study showed positive heterosis for most of the traits and over dominance for only some traits which corroborate the findings of Satenahalli et al. (1989) and Raju, (1990) which is ascribed to the allelic interaction.

The multiple trait evaluation of the twenty four hybrid combinations revealed that the combination MSO3 $\mathrm{x}$ APS45 ranked first followed by MSO1 x APS12. These observations confirm the established fact as observed by Ramesh Babu et al. (2002) that superiority of one or a couple of characters may not reflect the overall merit of the hybrid. Since the comprehensive merit of the hybrid over a range of traits depends on relative superiority of many individual traits, selection needs to be based on multiple traits contributing to overall silk output. Based on multiple traits and performance of the each of the hybrid combinations, the hybrids viz., MSO3 x APS45, MSO7 $\mathrm{x}$ APS8, MSO2 x APS45, MSO1 x APS12 and MSO1 x
APS45 were considered as potential hybrids and selected for further laboratory evaluation for the ultimate identification to the regions of tropical stress of Andhra Pradesh.

\section{References}

Datta RK, Nagaraju J (1987) Genetic engineering and tropical sericulture. Indian Silk 26, 9-12.

Eisen EJ, Horstgen-Schwark G, Saxton AM, Bandy TR (1983) Genetic interpretation and analysis of diallel crosses with animals. Theor Appl Genet 65, 17-23.

Falconer DS (1983) Introduction to quantitative genetics. Oliver and Boyd, Edinburgh, U.K.

Goel AK (2008) Breeding for the development of inbred lines and their heterotic effects on economic traits of silkworm, (Bombyx mori L.), Ph.D. thesis, University of Mysore, Mysore.

Griffing B (1956) Concepts on general and specific combining ability in relation to diallel crossing systems. Aust J Biol Sci 9, 463-493.

Harada C (1961) On the heterosis of quantitative characters in silkworm. Bull. Seric Expt Stn 17, 50-52.

Kempthorne O (1957) An Introduction to Genetic statistics. pp. 208 - 341, John Wiley and Sons, Inc, London.

Krishnaswami S, Narasimhann MN, Suryanarayana SK, Kumararaj S (1973) Silkworm rearing In: Manual on Sericulture, UN Food and Agriculture Organization, Rome, 2, 54-88.

Lakshmi H (2008) Studies on development of bivoltine hybrids of silkworm, Bombyx mori L. for tropical conditions of Andhra Pradesh. Ph.D. thesis, University of Mysore, Mysore.

Malik GN, Masoodi MA, Kamili AS, Sofi AM (1998) Studies on heterosis in some bivoltine silkworm (Bombyx mori L.) crosses. J Seric 6, 47-49.

Mano Y, Nirmal Kumar S, Basavaraja HK, Mal Reddy N, Datta RK (1993) A new method to select promising silkworm breeds / combinations. Indian Silk 31, 53.

Nanjundaswamy L (1997) Studies on the combining ability in silkworm, Bombyx mori L. Ph.D Thesis, University of Mysore, Mysore, India.

Raghavendra Rao D, Singh R, Premalatha V, Kariappa BK, Rekha M, Jayaswal KP (2002) Manifestation of hybrid vigour and combining ability in polyvoltine $\mathrm{x}$ bivoltine hybrid of silkworm, Bombyx mori L. Int J Indust Entomol 4, 23-30.

Raju PJ (1990) Studies on the hybridization and synthesis of new breeds of silkworm, Bombyx mori L. Ph.D. Thesis, University of Mysore, Mysore.

Ramesh Babu M, Chandrashekharaiah, Lakshmi H, Prasad J (2002) Multiple trait evaluation of bivoltine hybrids of silkworm (Bombyx mori L.). Int J Indust. Entomol 5, 37-43.

Rao NGP, Rana VKS, Tripati DP (1968) Line x Tester analysis 
of combining ability in sorghum. Ind $\mathrm{J}$ Genet and Plant Breeding, 28, 231-238.

Rao CGP, Chandrashekharaiah, Ibrahim Basha K, Seshagiri SV, Ramesh C, Nagaraju H (2004) Identification of superior polyvoltine hybrids (Poly x bivoltine) of silkworm, Bombyx mori L. Int J Indust Entomol 8, 43-49.

Ravindra Singh, Sharma SD, Raghavendra Rao D, Chandrashekaran K, Basavaraja HK, Kariappa BK (2005) Line x Tester and heterosis analysis in silkworm, Bombyx mori L. Indian J Seric 44, 92-99.

Satenahalli SB, Govindan R, Goud JV (1989) Heterosis studies for some quantitative traits in silkworm, Bombyx mori L. Indian J Seric 28, 100-102.

Seshagiri SV, Sankar Naik S, Goel AK, Raju PJ (2011) Identification of potential crossbreeds of mulberry silkworm,
Bombyx mori L. and their performance under tropical stress. Int J Indust Entomol 22, 45-49.

Shull GH (1909) A pure line method of corn breeding. Amer Breed Assoc. Rept. 5, 51-59.

Simmonds NW (1979) Principles of crop improvement. Longman Inc, New York.

Sudhakara Rao P, Datta RK, Basavaraja HK, Vijayakumari KM, Rekha M (2005) Evaluation of combining ability for certain quantitative traits through diallel crosses in the silkworm, Bombyx mori L. Indian J Seric 44, 75-81.

Toyama K (1905) Sericulture in Siam. Dainihon Sanshi Keinko pp. 24-26.

Yokoyama T (1979) Silkworm selection and hybridization. In: Genetics in relation to insect management. Working Papers. pp. 71- 83. The Rockfeller Foundation Management. 